\title{
Efeito clientela: uma análise da relação entre book-tax differences e dividendos extraordinários das empresas com ações listadas na Bovespa
}

\section{Clientele efect: an analysis of the relationship between book-tax differences and extraordinary dividends of companies listed in Bovespa}

\section{Efecto clientela: un análisis de la relación entre book-tax differences y dividendos extraordinarios de las empresas con acciones en la lista del Bovespa}

\section{Paulo Victor Gomes Novaes}

Mestre em Contabilidade e Finanças pela Universidade do Espírito Santo Professor Substituto da Graduação em Ciências Contábeis da Universidade do Espírito Santo Endereço: Avenida Fernando Ferrari, n॰ 514, Bairro Goiabeiras.

CEP: 29.075-910 - Vitória/ES - Brasil

E-mail: pvgnovaes@hotmail.com

Telefone: +55 (27) 4009-2794

\section{Alfredo Sarlo Neto}

Doutor em Contabilidade e Controladoria pela Universidade de São Paulo

Professor Adjunto da Universidade Federal do Espírito Santo

Endereço: Avenida Fernando Ferrari, n॰ 514, Bairro Goiabeiras.

CEP: 29.075-910 - Vitória/ES - Brasil

E-mail: sarloneto@ccje.ufes.br

Telefone: +55 (27) 4009-2794

\section{André Abreu de Almeida}

Doutorando em Administração pela Fundação Getúlio Vargas

Professor Assistente da Universidade Federal do Espirito Santo

Endereço: Avenida Fernando Ferrari, n॰ 514, Bairro Goiabeiras.

CEP: 29.075-910 - Vitória/ES - Brasil

E-mail: andre.a.almeida@ufes.br

Telefone: +55 (27) 4009-2794

\section{Luiz Cláudio Louzada}

Doutor em Administração pela Universidade Federal de Minas Gerais

Professor Assistente da Universidade Federal do Espirito Santo

Endereço: Avenida Fernando Ferrari, no 514. Bairro Goiabeiras.

CEP: 29.075-910 - Vitória/ES - Brasil

E-mail: andre.a.almeida@ufes.br

Telefone: $+55(27)$ 4009-2794

Artigo recebido em 18/11/2014. Revisado por pares em 27/07/2015. Reformulado em 17/08/2015. Recomendado para publicação em 27/08/2015 por Sandra Rolim Ensslin (Editora Científica). Publicado em 25/02/2016. 


\title{
Resumo
}

Este estudo objetivou verificar em que nível a existência de diferenças entre o lucro contábil e o lucro fiscal (Book-Tax Differences - BTDs) impacta significativamente a probabilidade de uma empresa propor o pagamento de dividendos adicionais. Embasado na metodologia empírico-analítica, com abordagem quantitativa, este estudo utilizou dados do Economatica® e a amostra consistiu em um conjunto de dados correspondentes ao período de 2010 a 2013, composto de empresas de capital aberto. Os resultados das regressões logísticas não permitiram confirmar a hipótese de que grandes diferenças causariam redução na chance de distribuição, o que revelou a preferência por distribuir dividendos, mesmo quando o lucro fiscal sobrepuja o lucro contábil (efeito clientela). Nos casos em que houve diferenças temporárias, a probabilidade de distribuição não é afetada, evidenciando que os gestores reconhecem que tais diferenças serão revertidas em um tempo próximo, por isso, não seria viável distribuir um lucro que carrega essas BTDs.

Palavras-chave: Book-Tax Differences (BTDs). Efeito Clientela. Dividendos Adicionais.

\begin{abstract}
This study aimed to verify at what level the existence of differences between accounting income and taxable income (Book-Tax Differences - BTDS) significantly impacts the likelihood of a company to propose the payment of additional dividends. Grounded in empirical-analytical methodology with a quantitative approach, this study used Economatica ${ }^{\circledR}$ data and the sample consisted of a set of data corresponding to the 2010-2013 period, composed of public companies. The results of logistic regressions did not allow to confirm the hypothesis that major differences would cause reduction in the chance of distribution, which revealed a preference for distributing dividends, even when tax profit surpasses the net income (clientele effect). In cases where there were temporary differences, the probability distribution is not affected, indicating that managers recognize that such differences will reverse in the near time, so it would not be feasible to distribute a profit carrying these BTDS.
\end{abstract}

Keywords: Book-Tax Differences (BTDs). Clientele effect. Additional dividends.

\section{Resumen}

Este estudio tenía el objetivo de verificar en qué nivel la existencia de diferencias entre el lucro contable y el lucro fiscal (Book-Tax Differences - BTDs) impacta significativamente la probabilidad de que una empresa proponga el pago de dividendos adicionales. Basado en la metodología empírico-analítica, con enfoque cuantitativo, este estudio utilizó datos del Economatica ${ }^{\circledR}$ y la muestra consistió en un conjunto de datos correspondientes al período de 2010 a 2013, compuesto de empresas de capital abierto. Los resultados de las regresiones logísticas no permitieron confirmar la hipótesis de que grandes diferencias causarían reducción en la chance de distribución, lo que reveló la preferencia por distribuir dividendos, aun cuando el lucro fiscal sobrepone el lucro contable (efecto clientela). En los casos en que hubo diferencias temporales, la probabilidad de distribución no es afectada, evidenciando que los gestores reconocen que tales diferencias serán revertidas en un tiempo próximo, por eso, no sería viable distribuir un lucro que carga esas BTDs.

Palabras clave: Book-Tax Differences (BTDs). Efecto Clientela. Dividendos Adicionales.

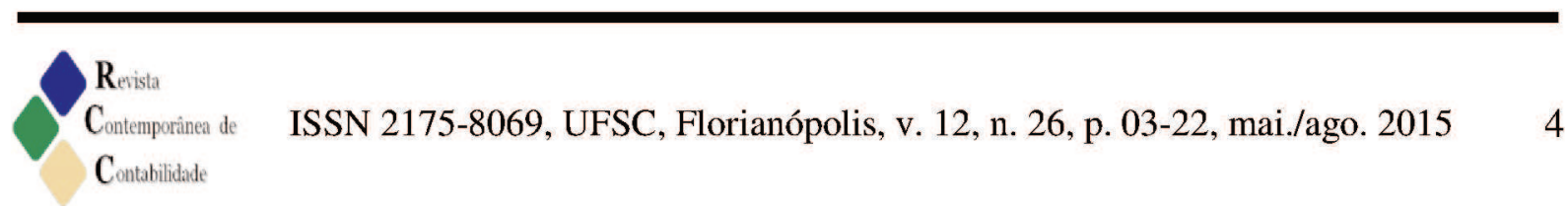




\section{Introdução}

O trade-off enfrentado pelos gestores, quando da apuração do tributo sobre o lucro, coloca em discussão as motivações para maximizar a riqueza do acionista e, ao mesmo tempo, minimizar o lucro tributável (PEREIRA, 2012). Nesse âmbito, tornaram-se comuns trabalhos que buscassem evidências da informatividade das diferenças entre o lucro apurado pela Contabilidade e aquele utilizado como base de cálculo para Imposto de Renda e Contribuição Social, consagradas como Book-Tax Differences - BTDs na literatura pertinente ao assunto.

Tang (2006) contribuiu para a literatura conferindo a relevância informacional das BTDs por meio do modelo de Ohlson. Já Ferreira et al. (2012) analisaram o tema relacionado ao gerenciamento de resultados, observados pelos accruals discricionários e observaram que as empresas gerenciam seus resultados na mesma direção do sinal das BTDs. Assim, sabidos que são fatores temporários e permanentes que motivam tais diferenças (DRAKE, 2013), torna-se coerente estudar o impacto que as decisões da administração podem causar nas deliberações da assembleia, considerando que tais ações dependem da análise de fluxo de caixa para serem realizadas, em especial no que tange à política de distribuição de riqueza.

Especificamente, a distribuição de dividendos foi regulamentada pela Lei n. 6.404/76, a qual impõe a distribuição obrigatória de um dividendo prioritário fixo ou mínimo, a ser consistentemente tratado no estatuto social. Isso foi complementado pela Lei n. 9.249/95 com a possibilidade de se cumprir esse obrigatório por meio de pagamento ou crédito dos chamados Juros Sobre Capital Próprio - JSCP (BRASIL, 1995).

Dessa forma, considerando o fato de a assembleia ter poder supremo sobre as deliberações quanto à distribuição de riqueza e constituições de reservas, é possível deliberar o pagamento de dividendos maiores ou menores do que aqueles previstos no estatuto, ponderando pela disponibilidade de fluxo de caixa por parte da empresa (Deliberação CVM n. 683/09).

Por outro lado, o seminal trabalho de Miller e Modigliani (1961) conduziu a interpretação de um fenômeno mercadológico chamado "efeito clientela". Eles explicam o acolhimento por parte das empresas à demanda constante pelo recebimento de dividendos, considerando a inexistência de um mercado perfeito.

A entrega da parte referente ao lucro auferido com os recursos dos acionistas é entendida como uma sinalização ao mercado tanto sobre o fluxo de caixa da empresa, quanto como uma resposta aos investidores no sentido de que seus recursos estão sendo bem administrados e estão gerando lucro (BHATTACHARYA, 1979).

Cabe ressaltar a atenção dada por Miller e Modigliani (1961, p. 431) ao dizerem que De todas as imperfeições que podem ser detalhadas [no mercado], a única que seria remotamente capaz de produzir certa concentração é a vantagem substancial de acordo com a capitalização de ganhos de capital quando comparada com os dividendos sob um ambiente com impostos (grifo dos autores).

Nesse sentido, é coerente restringir a análise do impacto das diferenças entre a Contabilidade e o Fisco no fluxo de caixa apenas daquelas empresas que propuseram distribuição do excesso de lucro na forma de dividendos adicionais. Assim, na tentativa de completar a lacuna existente na literatura sobre o trade-off de pagar dividendos adicionais e, com isso, atender aos anseios dos acionistas ou enxergar a redução do fluxo de caixa, causado pelas BTDs, este estudo se propõe a responder ao seguinte problema de pesquisa: Qual é a 
influência das Book-Tax Differences na proposição de pagamentos de dividendos adicionais para as empresas com ações negociadas na Bovespa?

Por isso, o objetivo geral é verificar em que nível a existência de BTDs impacta significativamente a probabilidade de uma empresa propor o pagamento de dividendos adicionais no mercado nacional, restrito ao processo de seleção da amostra. Ademais, objetiva-se também analisar se essa relação se mantém quando se controla por empresas que possuem grandes diferenças positivas (negativas) entre o lucro contábil e o lucro físcal. Ou ainda quando se controla pelo sinal da diferença.

A pesquisa contribui não só para a literatura no que diz respeito à política de dividendos e BTDs, mas também para o mercado de capitais, representado pelos investidores em geral, que poderão maximizar suas carteiras de investimentos melhorando suas restrições (em um caso específico de programação linear para formação de portfólios, por exemplo) e ainda conhecer o comportamento dos gestores quanto à proposição de distribuição do excesso de lucro. Investidores podem entrar ainda ingênuos no mercado e, então, ganharem experiência errando ou acompanhando erros alheios, ou podem buscar evidências como a que este artigo busca encontrar.

Assim, o trabalho está dividido em cinco partes, sendo esta primeira de caráter introdutório para apresentação do tema e do gap existente na literatura quanto a trabalhos dessa natureza; a segunda parte apresenta os principais fundamentos teóricos que embasarão as hipóteses a serem testadas e o cotejamento com os resultados; na terceira e na quarta parte, serão apresentados, respectivamente, o aspecto operacional e os resultados dos testes realizados, acompanhados de uma breve discussão; na quinta e última parte, são apresentadas as conclusões no intuito de verificar a aderência dos resultados às teorias do estudo.

\section{Referencial Teórico}

\subsection{Políticas de Dividendos}

A administração da empresa deve pensar na política de dividendos no intuito de atender à demanda de investidores de diferentes perfis. Enquanto alguns buscam remunerações mais altas na forma de dividendos, outros preferem optar por uma parcela maior dessa remuneração na forma de ganhos de capital; com isso, a preferência é por menores payouts ratio. Assim, a empresa se vê obrigada a proporcionar uma fatia do bolo que agrade medianamente a todos (BLACK; SCHOLES, 1974).

O que explica as diferentes demandas dos investidores está no fato de que um fundo de investimento, por exemplo, abarca pessoas juridicamente diferentes, isto é, dentre os poupadores, estão pessoas jurídicas normalmente tributadas, outras isentas, pessoas físicas e outros fundos de pensão, religiosos e de aposentadoria que também possuem benefícios fiscais sobre o pagamento de dividendos (BHATTACHARYA, 1979; BLACK; SCHOLES, 1974). Assim, é coerente pensar que cada um preferirá aquilo que mais lhe convém.

Por isso, Black e Scholes (1974), considerando o ambiente com diferentes impactos tributários, defenderam e comprovaram empiricamente a ideia de que o mercado é impulsionado, entre outras coisas, pelo que chamaram de "Efeito Incerteza". A teoria por eles levantada diz respeito à dualidade das hipóteses sobre o impacto da política de dividendos de uma empresa nos retornos ou nos preços de suas ações. 
Mas os autores fizeram uma ressalva quanto ao que chamaram de "Efeito Informacional", isto é, uma possível queda temporária no preço da ação referente à diminuição nos dividendos de uma empresa. Aos olhos do mercado, isso seria um sinal de "problemas em vista", mas que, caso não se concretizasse, logo os preços retornariam aos padrões normais (BLACK; SCHOLES, 1974).

Não obstante, apesar da falta de impacto permanente nos retornos e nos preços, a remuneração do capital próprio na forma de distribuição de dividendos funciona como uma sinalização do fluxo de caixa da empresa, reduzindo a assimetria de informações (BHATTACHARYA, 1979). Nessa linha, inspirados pela corrente positiva da Contabilidade, diversos trabalhos buscaram aproximar e explicar a relação entre números contábeis e o impacto no mercado de capitais, analisando a capacidade informacional dos dividendos (AHARONY; SWARY, 1980; ASQUITH; MULLINS, 1983; BRICKLEY, 1983; HEALY; PALEPU, 1988; KALAY; LOEWENSTEIN, 1985; LITZENBERGER; RAMASWAMY, 1979; MICHAELY; THALER; WOMACK, 1995).

No Brasil, Novis Neto e Saito (2002) encontraram evidências de retornos anormais das 90 cotações após o pagamento de dividendos. Percebeu-se um retorno anormal acumulado na ordem de $21,97 \%$ para as empresas que pagaram dividendos mais altos, de $5,16 \%$ para as companhias que pagaram dividendos intermediários e de $-15,50 \%$ para as empresas que pagaram dividendos mais baixos. Isso comprovou a teoria citada por Black e Scholes (1974) sobre o efeito temporário da informação, comprovada igualmente por Deangelo, Deangelo e Skinner (1996); Gonedes (1978); Penman (1983); e Watts (1973).

Partindo do entendimento que os dividendos mínimos são de certa forma conhecidos pelos investidores, por meio do estatuto social, Silva (2013) evidenciou que os dividendos adicionais propostos possuem uma carga informativa, detectada pelo modelo de Value Relevance, de Ohlson, aplicando-o às empresas brasileiras. Ressalta-se que esse é o único trabalho nacional publicado, até o momento, que trata especificamente dessa temática.

\subsubsection{Dividendos Adicionais Propostos}

Conforme o item 5 da Deliberação CVM n. 683/12, a assembleia é soberana em suas deliberações quanto à distribuição de dividendos, e assim é possível deliberar o pagamento de dividendos maiores ou menores do que aqueles previstos no estatuto. Cabe ressaltar que, em caso de omissão por parte do estatuto, o art. 202 da Lei n. 6.404/76 determina que os detentores de instrumentos de patrimônio (conforme o Pronunciamento Técnico CPC 39) não poderão receber um percentual inferior a $25 \%$ do lucro líquido ajustado.

Quando a decisão de distribuir o excesso de lucro acontece após o fim do exercício (e antes da aprovação das demonstrações), a obrigação não pode ser lançada direto no passivo, porque isso feriria o conceito de "obrigação presente na data das demonstrações contábeis", como definido na estrutura conceitual básica e no CPC 25 (Provisões, Passivos Contingentes e Ativos Contingentes). Por isso, eles devem ser divulgados em nota explicativa, como determina o CPC 26 (Apresentação das Demonstrações Contábeis).

\subsection{Efeito Clientela}

A principal teoria proposta pelo "mundo perfeito dos economistas" é que a política de dividendos não seria capaz de influenciar o valor da empresa. A ideia faz sentido, mas só é 
irrevogável quando se trata de um mercado perfeito, isto é, naquele em que não existem custos de transação, que a informação é disponível para todos de maneira simétrica e, principalmente, naquele onde não existem impostos atrelados aos negócios (MILLER; MODIGLIANI, 1961). No entanto, a realidade é que, apesar de as empresas divulgarem publicamente seus demonstrativos, isso não basta para que os resultados reflitam completamente o preço da ação (BEAVER, 2002).

Embora pesquisas empíricas tenham relatado comportamentos que vão ao encontro da eficiência do mercado de capitais em meados do século XX (BALL; BROWN, 1968; BEAVER, 1968; ARCHIBALD, 1972), outros estudos revelaram que isso não é uma realidade imutável (ANTUNES; LAMOUNIER; BRESSAN, 2006). Com isso, percebe-se que "o mercado eficiente também afeta as interpretações dos pesquisadores dando lugar a associações observadas entre os preços das ações e os números contábeis" (BEAVER, 2002, p. 454).

No âmbito da remuneração de capital, Miller e Modigliani (1961, p. 432) contribuem dizendo que o diferencial do imposto que conduz aos ganhos de capital é "indubitavelmente a maior imperfeição sistemática do mercado". Dessa forma, eles entendem que, para a maioria dos detentores de instrumento patrimonial, a melhor saída é optar por receber dividendos em detrimento dos ganhos de capital.

Assim, o ponto principal sobre política de dividendos é que as imperfeições do mercado "podem levar um investidor a ter uma preferência sistemática por um dólar em dividendos correntes em relação a um dólar na forma de ganho de capital" (MILLER; MODIGLIANI, 1961, p. 431). Com isso, considerando as imperfeições do mercado, o efeito clientela é explicado pela suposta preferência de uma parte dos investidores em receber pagamentos de dividendos, em vez de ganhos de capital (NOVIS NETO; SAITO, 2002).

\subsection{Book-Tax Differences e Dividendos}

A forma específica como o fisco exige que seja calculada sua fatia no bolo (na forma de tributos) vai de encontro ao postulado contábil da competência. Sem ele, o modelo contábil é invalidado e a contabilidade perderia sua função de medida de desempenho e se minimizaria a uma análise financista de fluxo de caixa (WATTS; ZIMMERMAN, 1978).

Naturalmente, esse encontro provoca diferenças nos valores de receitas, despesas e, consequentemente, no lucro. A essa diferença a literatura internacional deu o nome de booktax differences, que são, portanto, as diferenças entre o lucro apurado pela contabilidade e aquele utilizado para fins fiscais (HANLON, 2005).

No entanto, sabe-se que existem diferentes fatores que formam as BTDs. Drake (2013) investigou se os estágios do ciclo de vida impactam na relação entre as BTDs e a persistência dos lucros, relação esta que fora apresentada por Hanlon (2005) no sentido de que grandes diferenças, tanto para cima quanto para baixo, estão relacionadas à maior variabilidade dos lucros, o que é considerado ruim para o mercado, que não consegue precificar corretamente o valor de ação de uma empresa nessa situação (DECHOW; GE; SCHRAND, 2010).

Seguindo esse estudo, outros trabalhos demonstraram a capacidade informacional das BTDs no mercado de capitais. Joos, Pratt e Young (2000) evidenciaram que os retornos das ações das empresas americanas são menos associados aos lucros quando as diferenças (BTDs) são grandes e voláteis. Dechow, Ge e Schrand (2010) demonstraram empiricamente que lucros menos voláteis indicam melhores inputs para os modelos de avaliação de empresa, a

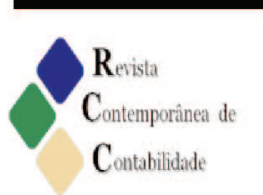

ISSN 2175-8069, UFSC, Florianópolis, v. 12, n. 26, p. 03-22, mai./ago. 2015 
exemplo do modelo de Feltham-Ohlson, e, dessa forma, lucros mais persistentes agregam mais qualidade do que lucros menos persistentes.

Contudo, a atenção dada a Drake (2013) se justifica pela análise dos fatores permanentes e temporários que compõem as diferenças, analisadas nos quartis extremos, isto é, aqueles valores pertencentes ao primeiro e ao quarto quartis, no intuito de captar unicamente diferenças grandes. O Quadro 1 evidencia algumas fontes de diferenças entre as orientações de melhores práticas apresentadas pelos Pronunciamentos Técnicos da Contabilidade Societária e as imposições tributárias:

\section{Quadro 1 - Fontes de diferenças entre a Contabilidade Societária e a Tributária}

\begin{tabular}{|c|c|}
\hline $\begin{array}{l}\text { FONTES DE } \\
\text { DIFERENÇAS }\end{array}$ & SOCIETÁRIA \\
\hline Imobilizado & $\begin{array}{ll}\text { Em geral, o padrão contábil orienta a } & \text { A norma físcal permite a depreciação } \\
\text { acelerada com base no limite de vida útil } \\
\text { depreciação dos Ativos com base na vida útil } \\
\text { econtipulada pelo regulamento de imposto de } \\
\text { econda (RIR/99) }\end{array}$ \\
\hline Receitas & 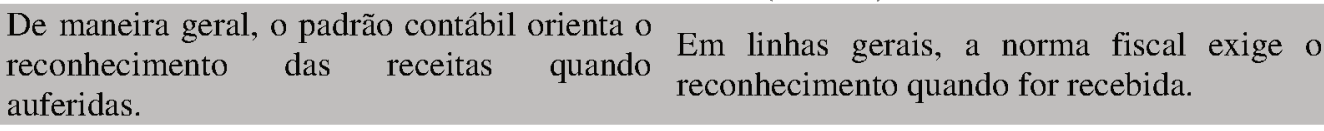 \\
\hline Estoques & 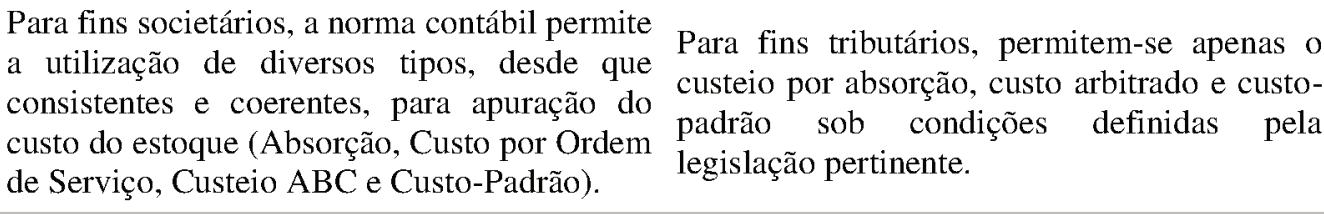 \\
\hline $\begin{array}{l}\text { Provisões para } \\
\text { Ajustes a Valor } \\
\text { de Mercado }\end{array}$ & $\begin{array}{ll}\text { ntábil orienta a redução ao valor } & A \\
\text { dos estoques (CPC 27) } & \text { um }\end{array}$ \\
\hline
\end{tabular}

Fonte: Adaptado de Drake (2013).

Com isso, percebe-se que as diferenças provocadas pelas imposições da contabilidade tributária refletem na contabilidade societária, para as empresas tributadas com base no lucro real, na forma das adições e exclusões, que devem ser devidamente registradas na "Parte A" do Livro de Apuração do Lucro Real - LALUR. Cabe ressaltar que o advento da Lei n. 12.973/2014 fará com que novas diferenças existam entre a contabilidade societária e a tributária. No entanto, antecipa-se que tais impactos não serão percebidos no período amostral deste estudo. Para melhor visualização da segregação dos fatores temporários e permanentes, apresenta-se o Quadro 2.

\section{Quadro 2 - Diferenças temporárias e permanentes}

\begin{tabular}{|c|c|}
\hline Adições & Exclusões \\
\hline Temporárias & Temporárias \\
\hline o Provisão para contingências & o Depreciação acelerada incentivada \\
\hline o Despesas pré-operacionais & o Ganhos cambiais (tributados em regime de caixa) \\
\hline \multicolumn{2}{|l|}{ o Perdas Cambiais (tributadas em regime de caixa) } \\
\hline Permanentes & Permanentes \\
\hline o Multas & o Receitas de Incentivos Fiscais \\
\hline o Resultado Negativo de Equivalência Patrimonial & o Resultado Positivo de Equivalência Patrimonial \\
\hline o Depreciação da Mais Valia ("Demmed Cost") & \\
\hline
\end{tabular}

Fonte: PricewaterhouseCoorpers (2011). 


\subsection{Hipóteses}

Com base na teoria abordada, espera-se que empresas com grandes diferenças entre a contabilidade societária e a tributária diminuam a propensão a propor o pagamento de dividendos adicionais. Assim, especificamente, a hipótese 1 é constituída dessa forma:

$\boldsymbol{H}_{\boldsymbol{I}}$ - A probabilidade da proposição de pagamento de dividendos adicionais das empresas pertencentes aos quartis extremos, grande positivo (GP) e grande negativo (GN), tenderá a ser menor em relação aos quartis medianos.

Este estudo visa investigar os desdobramentos das diferenças causadas pela fiscalidade no âmbito contábil. Isso é, de maneira específica, a parte negativa das BTDs temporárias e permanentes. Por causa disso, sabendo que uma diferença negativa induz a um pagamento de imposto proporcionalmente maior em relação ao lucro contábil divulgado, constitui-se a principal hipótese deste estudo:

$\boldsymbol{H}_{2}$ - Devido ao impacto causado pelas BTDs no fluxo de caixa, controlando pelas diferenças negativas, espera-se que a probabilidade de as empresas proporem o pagamento de dividendos adicionais será menor em relação àquelas com diferenças positivas.

Adicionalmente, serão testados os desdobramentos das BTDs, observando, de forma isolada, as diferenças temporárias e permanentes. Ainda, seus grupos extremos, ou seja, as empresas classificadas no $1^{\circ}$ e no $4^{\circ}$ quartis, de acordo com a magnitude das diferenças.

\section{Metodologia}

Embasado na metodologia empírico-analítica, com abordagem quantitativa, este estudo se propõe explorar e analisar a influência das diferenças entre o lucro contábil e o lucro fiscal (Book-tax differences) na propensão à proposição do pagamento de dividendos adicionais.

\subsection{Amostra}

Os dados foram coletados no banco de dados do Economática, e a amostra consiste em um conjunto de dados empilhados, correspondente ao período de 2010 a 2013, composto de empresas de capital aberto, com ações negociadas na Bovespa e que passaram pelo processo de seleção da amostra, conforme a Tabela 1 .

Tabela 1 - Processo de seleção da amostra

\begin{tabular}{|c|c|c|}
\hline SELEÇÃO DA AMOSTRA & & $\mathbf{N}^{0}$ Obs. \\
\hline \multicolumn{3}{|l|}{ Seleção das empresas que distribuíram dividendos adicionais } \\
\hline Todas as empresas com dados disponíveis no Economática & & 1452 \\
\hline Exclusão das empresas que não propuseram dividendos adicionais & -1189 & 263 \\
\hline Exclusão das Empresas sem informação de Valor de Mercado & -29 & 234 \\
\hline Exclusão das Empresas que não apresentaram IR Diferido & -20 & 214 \\
\hline Exclusão das Empresas com Dividendos Adicionais propostos negativos & -3 & 211 \\
\hline \multicolumn{3}{|l|}{ Seleção das empresas que não distribuíram dividendos adicionais } \\
\hline Todas as empresas que distribuíram dividendos adicionais no período & & 1189 \\
\hline Exclusão das Empresas com valor diferente de zero para as variáveis dos modelos & -649 & 540 \\
\hline Exclusão com base na seleção aleatória do mesmo nºbservações da seleção & -329 & 211 \\
\hline
\end{tabular}

Fonte: Dados da Pesquisa.

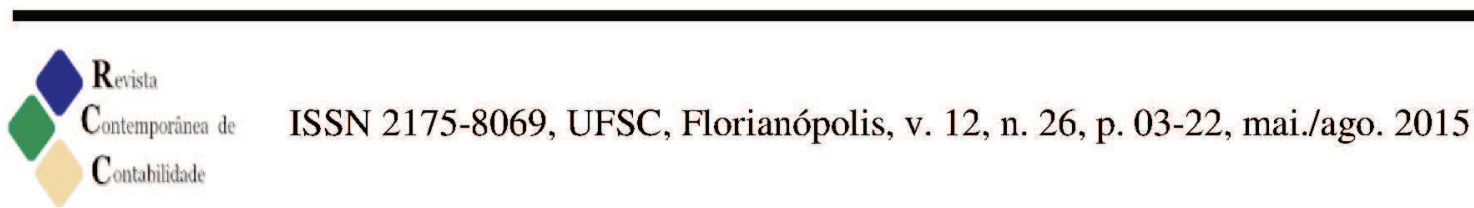


No intuito de balancear a amostra para buscar mais acurácia do modelo de regressão logística, isto é, equilibrar o número de empresas que atendem ao evento de interesse em relação àquelas que não atendem, extraiu-se uma segunda amostra apenas com as empresas que não propuseram o pagamento de dividendos adicionais, seguindo as mesmas características setoriais observadas na primeira amostra, chegando à Tabela 2 .

TABELA 2 - Classificação das amostras entre os setores

\begin{tabular}{ll|ll|l|l|l|l|l|l|l|l}
\hline \multirow{2}{*}{ Código } & Setores & $\mathbf{2 0 1 0}$ & $\mathbf{2 0 1 1}$ & $\mathbf{2 0 1 2}$ & $\mathbf{2 0 1 3}$ & & Total Geral \\
\hline 1 & Sim & Não & Sim & Não & Sim & Não & Sim & Não & Sim & Não \\
\hline 2 & Alimentos e Bebidas & 3 & 3 & 2 & 2 & 2 & 2 & 3 & 3 & 10 & 10 \\
3 & Comércio & 6 & 6 & 5 & 5 & 7 & 6 & 5 & 6 & 23 & 23 \\
4 & Construção & 1 & 1 & 1 & 1 & 0 & 0 & 1 & 1 & 3 & 3 \\
5 & Energia Elétrica & 17 & 12 & 17 & 11 & 13 & 13 & 16 & 11 & 63 & 47 \\
6 & Máquinas Indust. & 2 & 1 & 1 & 2 & 1 & 1 & 1 & 1 & 5 & 5 \\
7 & Minerais não Met. & 0 & 0 & 0 & 0 & 0 & 0 & 1 & 1 & 1 & 1 \\
8 & Outros & 8 & 12 & 9 & 15 & 10 & 10 & 9 & 15 & 36 & 52 \\
9 & Papel e Celulose & 2 & 2 & 1 & 1 & 2 & 2 & 2 & 2 & 7 & 7 \\
10 & Petróleo e Gás & 0 & 0 & 0 & 0 & 0 & 0 & 1 & 1 & 1 \\
11 & Química & 1 & 1 & 2 & 2 & 1 & 1 & 3 & 3 & 7 \\
12 & Siderurgia \& Metal & 3 & 3 & 2 & 2 & 2 & 2 & 2 & 2 & 9 \\
13 & Software e Dados & 0 & 1 & 0 & 1 & 1 & 0 & 2 & 1 & 3 \\
14 & Telecomunicações & 0 & 0 & 1 & 1 & 2 & 2 & 1 & 1 & 4 \\
15 & Têxtil & 4 & 4 & 3 & 3 & 6 & 6 & 4 & 4 & 17 \\
16 & Transporte Serviços & 2 & 2 & 2 & 2 & 2 & 2 & 2 & 2 & 8 & 8 \\
17 & Veículos e peças & 3 & 3 & 4 & 4 & 3 & 3 & 3 & 3 & 13 \\
\hline
\end{tabular}

Nota: "Sim" equivale à amostra das empresas que atendem ao evento de interesse (distribuição de dividendos adicionais), e "não" para a amostra das empresas que não propuseram.

Ressalta-se que não foi possível selecionar o mesmo número de empresas, principalmente para o setor de Energia Elétrica. Dessa forma, optou-se por preencher a quantidade de empresas faltantes naquelas características com o setor "Outros". Como critério de seleção da segunda amostra, utilizou-se a função "aleatórioentre" do software Microsoft Excelß, seguido de uma coleta manual para cada setor e para cada ano.

\subsection{Variável Dependente}

O modelo proposto pretende explicar a variação que as diferenças entre a contabilidade societária e a tributária causam na probabilidade de distribuição dos dividendos adicionais $\left(\boldsymbol{D} \boldsymbol{I} \boldsymbol{V}_{i t}\right)$, que representam a variável dependente do modelo de regressão logística. Assim, essa variável dummy assumirá o valor 1 se a empresa i propôs a distribuição de dividendos adicionais no tempo t; e 0 , caso contrário. 


\subsection{Variáveis de Interesse}

Considerando que o intuito do modelo é capturar os desdobramentos das BTDs, não haverá apenas uma variável de interesse. Logo, de maneira geral, a Equação 1 demonstra o cálculo das diferenças totais:

$$
B T D_{t}=L C_{i t}-L F_{i t}
$$

Em que: $\boldsymbol{B} \boldsymbol{T} \boldsymbol{D}_{\boldsymbol{t}}=$ Diferenças entre o Lucro Contábil e o Lucro Fiscal; $\boldsymbol{L} \boldsymbol{C}_{i t}=$ Lucro apurado pelos critérios da contabilidade; e $\boldsymbol{L F}_{i t}=$ Lucro apurado para fins de pagamento de imposto de renda e contribuição social.

Já a Equação 2 demonstra o cálculo da estimativa de Lucro Fiscal:

$$
L F_{i t}=\frac{I R_{-} C S_{i t}}{0,34}
$$

Em que: $\boldsymbol{I R} \boldsymbol{C S}_{i t}$ equivale à despesa corrente de IR/CS a pagar no tempo t. Ressalta-se que por falta de informações sobre o Livro de Apuração do Lucro Real (LALUR), utilizar-seá como estimativa geral para taxa de imposto de renda e contribuição social para todas as empresas a taxa fixada em $34 \%$ (15\% de IR + 10\% IR Adicional + 9\% de CSLL), mesmo reconhecendo a existência de padrões setoriais que fogem a essa regra. Dessa maneira, uma vez que se sabe que é possível estimar as diferenças temporárias, por meio da Equação 3:

$$
B T D T_{i t}=\frac{I R_{-} D I F_{i t}}{0,34}
$$

Em que: $\boldsymbol{B} \boldsymbol{T} \boldsymbol{D} \boldsymbol{T}_{\boldsymbol{i t}}=$ Diferenças advindas de fatores temporários. $\boldsymbol{I} \boldsymbol{R}_{-} \boldsymbol{D} \boldsymbol{I} \boldsymbol{F}_{\boldsymbol{i t}}=$ Imposto de Renda Diferido captado pela Demonstração do Resultado para que uma só variável apresente ambos os sinais, pois as diferenças podem tanto ser positivas quanto negativas.

A diferença positiva é causada quando o imposto que é pago em um período era devido para fins fiscais, mas não o era para fins societários. Por isso, o valor deve ser controlado no grupo de Impostos Diferidos dentro do Ativo até a chegada de sua competência para que, então, o valor seja reconhecido como corrente (PRONUNCIAMENTO TÉCNICO CPC 32). De maneira contrária, no grupo de mesmo nome, porém no passivo, ficam os valores devidos societariamente, mas não ainda tributariamente, causando uma diferença negativa entre o lucro contábil (considerando apenas esta operação) e o lucro fiscal.

Uma vez conhecidos os impactos temporários nas BTDs, é possível estimar a Equação 4:

$$
B T D P_{i t}=B T D_{i t}-B T D T_{i t}
$$

Dessa maneira, estimam-se os fatores permanentes na formação das diferenças totais. Trata-se de receitas e despesas adicionadas ou excluídas não por uma questão de timing, mas, sim, por indedutibilidade ou não tributação de forma definitiva.

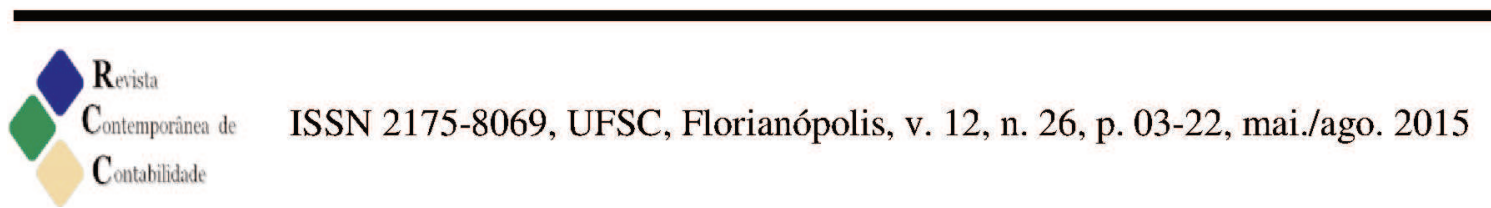




\subsection{Variáveis de Controle}

Segundo Fávero et al. (2009), as variáveis de controle possuem uma função que vai além de exploratória em uma função de regressão. Elas servem para isolar o efeito dos coeficientes das variáveis de interesse sobre a variável dependente (BTDs). Para efeito de visualização nos modelos econométricos, as variáveis de controle aparecerão resumidas no símbolo $\Sigma_{j}^{k} V C_{t}$.

Dessa forma, dando ênfase aos resultados empíricos nacionais, considerando que nem sempre é possível comparar que o comportamento seja similar aos diagnosticados no mercado americano, serão testadas as variáveis:

- Endividamento - Segundo Abreu (2004), tende a ensejar uma relação negativa, pois empresas a alavancagem de uma empresa naturalmente diminuirá ou extinguirá o excesso de lucro; logo, espera-se que haverá redução na proposição de distribuição de dividendos adicionais.

- Tamanho - Utilizando como proxy o logaritmo natural do Ativo Total no final do ano, espera-se que empresas maiores distribuam mais dividendos adicionais, considerando a concentração do capital, reconhecidamente presente no mercado brasileiro, o que provoca o efeito alinhamento.

\subsection{Regressões Logísticas}

A regressão logística, ou logit, é uma técnica estatística usada para investigar o efeito de variáveis explicativas métricas ou não métricas sobre probabilidade de ocorrência de sucesso em uma variável dependente necessariamente qualitativa. Ou seja, é quando a variável a ser explicada é representada por uma dummy que assume o valor 1 para o sucesso, e 0 para o insucesso (FÁVERO et al., 2009).

Nesta pesquisa, sucesso é o caso em que a empresa propôs a distribuição de dividendos adicionais, e insucesso quando não o propôs. O esquema (Figura 1) demonstra os desdobramentos do modelo de modo a facilitar o entendimento:

Figura 1 - Desdobramentos do modelo econométrico

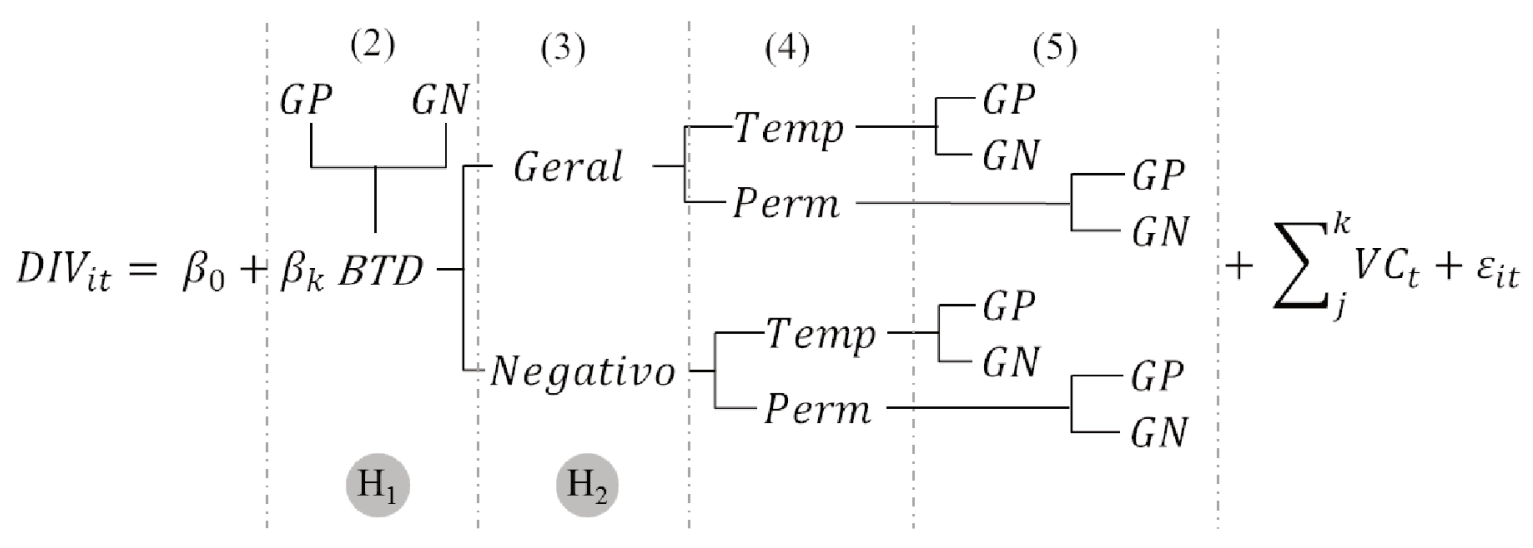

13 ISSN 2175-8069, UFSC, Florianopolis, v. 12, n. 26, p. 03-22, mai./ago. 2015 
O modelo geral (1) regredirá as diferenças totais no período t contra a proposição do pagamento de dividendos adicionais, além das variáveis de controle $\sum_{j}^{k} V C_{t}$ (presentes em todos os modelos). O modelo (2) acrescenta a interação das BTDs com duas variáveis dummy que assumem o valor 1 quando pertencerem aos quartis 1 e 4 , no intuito de capturar as grandes diferenças, positivas (GP) e negativas (GN).

O modelo (3) busca isolar as diferenças negativas, com a inclusão de uma variável dummy interagida com a magnitude da diferença. O modelo seguinte (4) analisará a composição das diferenças em temporárias e permanentes. Isso será especificado no modelo (5), que, por fim, busca captar os efeitos dos desdobramentos do modelo anterior, controlando as grandes diferenças positivas e negativas em cada tipo de diferença.

\section{Apresentação e Discussão dos Resultados}

\subsection{Estatística Descritiva e Correlação de Pearson}

Utilizando o pacote estatístico Stata® e o software Microsoft Excel, com base nos procedimentos relatados no tópico anterior, apresentam-se as estatísticas descritivas na Tabela 3.

Tabela 3 - Estatísticas Descritivas

\begin{tabular}{|c|c|c|c|c|c|c|c|}
\hline AMOSTRA & VARIÁVEL & OBS & MÉDIA & MEDIANA & DESV.PAD & MIN & MAX \\
\hline \multirow{6}{*}{ 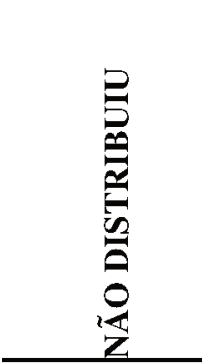 } & BTD_TOT & 211 & 35938.29 & 17655.12 & 381520.36 & -2165946.59 & 2678544.88 \\
\hline & BTDTOT_NEG & 211 & -81352.88 & 0.00 & 237272.55 & -2165946.59 & 0.00 \\
\hline & BTD_TEMP & 211 & 28863.58 & 1870.59 & 328511.62 & -1296623.53 & 2791091.18 \\
\hline & BTD_PERM & 211 & 7074.71 & 6766.12 & 303209.50 & -1673905.59 & 2207746.59 \\
\hline & TAM & 211 & 14.82 & 14.95 & 1.43 & 10.68 & 18.07 \\
\hline & ENDIV & 211 & 0.61 & 0.61 & 0.21 & 0.03 & 1.30 \\
\hline \multirow{6}{*}{ 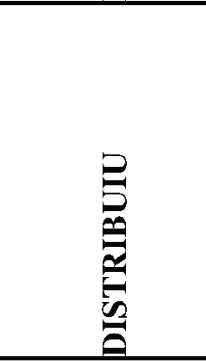 } & BTD_TOT & 211 & 293898.31 & 66570.76 & 1086035.32 & -2096411.76 & 8187531.06 \\
\hline & BTDTOT_NEG & 211 & -48801.04 & -48801.04 & 204712.84 & -2096411.76 & 0.00 \\
\hline & BTD_TEMP & 211 & 14278.02 & 14278.02 & 557491.81 & -4044117.65 & 2500702.94 \\
\hline & BTD_PERM & 211 & 279620.29 & 279620.29 & 856519.14 & -341152.47 & 6583407.53 \\
\hline & TAM & 211 & 15.21 & 15.21 & 1.44 & 12.25 & 19.71 \\
\hline & ENDIV & 211 & 0.52 & 0.52 & 0.20 & 0.03 & 1.13 \\
\hline \multirow{6}{*}{$\frac{i}{\frac{\pi}{c}}$} & BTD_TOT & 422 & 164918.30 & 39126.09 & 823174.91 & -2165946.60 & 8187531.10 \\
\hline & BTDTOT_NEG & 422 & -65076.96 & 0.00 & 221927.25 & -2165946.60 & 0.00 \\
\hline & BTD_TEMP & 422 & 21570.80 & 2383.82 & 457071.68 & -4044117.70 & 2791091.20 \\
\hline & BTD_PERM & 422 & 143347.50 & 31892.50 & 656059.77 & -1673905.60 & 6583407.50 \\
\hline & TAM & 422 & 15.01 & 15.04 & 1.44 & 10.68 & 19.71 \\
\hline & ENDIV & 422 & 0.56 & 0.58 & 0.21 & 0.03 & 1.30 \\
\hline
\end{tabular}

ISSN 2175-8069, UFSC, Florianópolis, v. 12, n. 26, p. 03-22, mai./ago. $2015 \quad 14$

Contemporianea de

Contabilidide 
(i) BTD_TOT = Diferenças totais entre o lucro contábil e o lucro fiscal na empresa no final do ano; (ii) BTDTOT_NEG = Variável que captura apenas as diferenças negativas; (iii) $\mathbf{B T D} \_$TEMP $=$Variável que captura apenas as diferenças temporárias; (iv) BTD_PERM = Variável que captura apenas as diferenças permanentes (v) TAM = Tamanho da empresa representado pelo log do Ativo Total da empresa no final do ano; e (vi) ENDIV = Índice de Endividamento da empresa no final do ano.

Percebe-se uma dispersão bastante acentuada na variável BTD_TOT na amostra geral. Cabe ressaltar que é entre as empresas que distribuem dividendos adicionais que essa variabilidade é ainda maior. E mais, as empresas que propuseram o pagamento de dividendos adicionais apresentaram, em média, 8.18 vezes mais em diferenças totais entre o lucro contábil e o lucro fiscal. Essa diferença é ainda maior quando se analisam exclusivamente as diferenças permanentes, que foram quase 40 vezes maiores naquelas empresas que distribuíram dividendos adicionais.

Por outro lado, analisando apenas as diferenças negativas, isto é, quando o lucro fiscal passa a ser maior do que o contábil, em média, as empresas que não propuseram o pagamento de dividendos adicionais apresentaram diferenças $67 \%$ maiores. Já nas diferenças temporárias, observou-se o dobro do valor nas empresas que não atenderam ao evento de interesse, porém com uma dispersão também maior.

Por fim, observou-se também que empresas que não distribuíram dividendos adicionais apresentam, em média, um índice de endividamento $8 \%$ menor do que as empresas que os distribuíram.

Com a justificativa de que a variável que captura as diferenças totais naturalmente estaria correlacionada com seus desdobramentos, a Tabela 4 apresenta a matriz de correlação de Pearson apenas para algumas variáveis.

Tabela 4 - Matriz de correlação de Pearson

\begin{tabular}{|c|c|c|c|c|}
\hline & BTD_TOT & BTD_TEMP & BTD_PERM & TAM \\
\hline \multicolumn{5}{|l|}{ BTD_TOT } \\
\hline BTD_TEMP & $0.6061 * * *$ & & & \\
\hline BTD_PERM & $0.8324 * * *$ & 0.0638 & & \\
\hline TAM_1 & $0.2598 * * *$ & 0.01 & $0.3190 * * *$ & \\
\hline ENDIV & $-0.1183^{* * *}$ & $-0.083 *$ & $-0.0906^{*}$ & $0.2805 * * *$ \\
\hline
\end{tabular}

(i) Sobre os asteriscos, ${ }^{* * *} \mathrm{p}<0.01$, ${ }^{*} * \mathrm{p}<0.05, \mathrm{e}^{*} \mathrm{p}<0.10$; (ii) BTD_TOT $=$ Diferenças totais entre o lucro contábil e o lucro fiscal na empresa no final do ano; (iii) BTD_TEMP = Variável que captura apenas as diferenças temporárias; (iv) BTD_PERM = Variável que captura apenas as diferenças permanentes (v) TAM = Tamanho da empresa representado pelo log do Ativo Total da empresa no final do ano; e (vi) ENDIV = Índice de Endividamento da empresa no final do ano.

O coeficiente de correlação mede o grau de associação entre duas variáveis padronizadas, variando, portanto, entre -1 (negativa perfeita) e 1 (positiva perfeita). Dessa forma, percebe-se a inexistência de correlação alta entre as variáveis explicativas, exceto entre as variáveis BTD_TOT e BTD_PERM, que apresentaram um coeficiente de correlação acima de 0,8 , o que é considerado alto (LEVINE, 2012). No entanto, elas não são dispostas conjuntamente em nenhum dos modelos. O que se depreende, nesse caso, é a preponderância 
das diferenças permanentes em relação ao total. Ademais, observa-se a inexistência de relação entre as diferenças permanentes e as temporárias.

Além disso, observa-se o sinal negativo na relação entre as BTDs e Endividamento, indicando, a priori, que empresas mais endividadas apresentam menores diferenças entre o lucro contábil e o fiscal, ao passo que empresas maiores apresentam BTDs também maiores. Na relação entre tamanho e endividamento, percebe-se que empresas maiores tendem a ficar mais endividadas, dado o coeficiente significativo e positivo $(r=0.2805)$.

\subsection{Discussão dos Resultados}

Os modelos indicados buscam verificar a influência das diferenças entre o lucro contábil e o lucro fiscal na probabilidade de a empresa ter indicado a distribuição de dividendo adicional. Ressalta-se que o modelo padrão (1) está embasado pela formulação teórica supramencionada, enquanto os demais modelos referem-se a desdobramentos do primeiro, com o propósito de investigar possíveis características não observáveis, em princípio. Assim, a Tabela 6 apresenta os resultados dos cinco modelos de regressão logística.

$$
\begin{gathered}
D I V_{i t}=\beta_{0}+\beta_{1} B T D_{-} T O T_{i t}+\sum_{j}^{k} V C_{t}+\varepsilon_{i t} \\
D I V_{i t}=\beta_{0}+\beta_{1} G N B T D_{i t}+\beta_{2} G P B T D_{i t}+\sum_{j}^{k} V C_{t}+\varepsilon_{i t} \\
D I V_{i t}=\beta_{0}+\beta_{1} B T D G E R A L_{i t}+\beta_{2} B T D_{-} N E G_{i t}+\sum_{j}^{k} V C_{t}+\varepsilon_{i t}
\end{gathered}
$$

$D I V_{i t}=\beta_{0}+\beta_{1} B T D T E M P_{-} G E R A L_{i t}+\beta_{3}$ BTDPERM_GERAL $L_{i t}+\beta_{2}$ BTDTEMP_NEG $G_{i t}+$

$\beta_{4} B T D P E R M_{-} N E G_{i t}+\sum_{j}^{k} V C_{t}+\varepsilon_{i t}$

$D I V_{i t}=\beta_{0}+\beta_{1} G N_{-} B T D T E M P_{-} G E R A L_{i t}+\beta_{2} G P_{-} B T D T E M P_{-} G E R A L_{i t}+$

$\beta_{3} G N_{-} B T D P E R M_{-} G E R A L_{i t}+\beta_{4} G P P_{-} B T D P E R M_{-} G E R A L_{i t}+\beta_{5} G N_{-} B T D T E M P_{-} N E G_{i t}+$

$\beta_{6} G P_{-} B T D T E M P_{-} N E G_{i t}+\beta_{7} G N_{-} B T D P E R M_{-} N E G_{i t}+\beta_{8} G P_{-} B T D P E R M_{-} N E G_{i t}+$

\begin{tabular}{|c|c|c|c|c|c|}
\hline VARIÁVEIS & (1) & (2) & (3) & (4) & (5) \\
\hline BTD_TOT & $\begin{array}{c}* \\
1.0000\end{array}$ & & 1.0000002 & & \\
\hline GNBTD_TOT & & $\begin{array}{c}* \\
1.000001\end{array}$ & & & \\
\hline GPBTD_TOT & & 1.0000002 & & & \\
\hline
\end{tabular}
$\sum_{j}^{k} V C_{t}+\varepsilon_{i t}$

Tabela 5 - Regressões logísticas

BTDTOT_NEG

1.0000008

BTD_TEMP 
BTD PERM

1.0000003

\begin{tabular}{cc} 
BTDTEMP_NEG & 1.0000003 \\
BTDPERM_NEG & $* * *$ \\
\hline
\end{tabular}

GNTEMP

0.9999998

GPTEMP

1.0000002

GNTEMPNEG

1.0000

GPTEMPNEG

0.9999331

GNPERM

***

1.000022

GPPERM

1.0000001

$* *$

GN_PERMNEG (NEG)

0.999987

\section{GP_PERMNEG}

\begin{tabular}{|c|c|c|c|c|c|}
\hline & $* * *$ & $* * * *$ & $* * * *$ & $* * * *$ & $* * *$ \\
\hline I AlM_I & 1.352561 & 1.426181 & 1.427608 & 1.514371 & 1.540335 \\
\hline \multirow{2}{*}{ ENDIV (NEG) } & $* * * *$ & $* * * *$ & $* * *$ & $* * *$ & $* * *$ \\
\hline & 16.4611 & 17.63702 & 17.63702 & 17.236 & 14.95432 \\
\hline \multirow{2}{*}{ CONSTANT (NEG) } & $* * * *$ & $* * * *$ & $* * * *$ & $* * * *$ & $* * * *$ \\
\hline & 20.34835 & $\mathbf{4 0 . 4 8 7 7 7}$ & 41.59583 & 88.32295 & 114.0914 \\
\hline OBSERVAÇÕES & 422 & 422 & 422 & 422 & 422 \\
\hline PSEUDO R ${ }^{2}$ & $7.57 \%$ & $7.80 \%$ & $7.79 \%$ & $13.10 \%$ & $14.60 \%$ \\
\hline QUI-QUADRADO & 44.31 & 45.64 & 45.56 & 76.58 & 85.17 \\
\hline
\end{tabular}

Em que: (i) BTD_TOT = Diferenças totais entre o lucro contábil e o lucro fiscal na empresa no final do ano; (ii) GNBTD_TOT representa as diferenças totais localizadas no primeiro quartil (grande negativo); (iii) BTD_PERM = Variável que captura apenas as diferenças permanentes; (iv) BTDPERM_NEG = Variável que captura apenas as diferenças permanentes negativas; (v) GNPERM = Variável que captura apenas as diferenças permanentes localizadas no primeiro quartil; (vi) GNPERM_NEG = Variável que captura apenas as diferenças permanentes negativas localizadas no primeiro quartil; (vii) GPPERM_NEG = Variável que captura apenas as diferenças permanentes localizadas no último quartil (grande positivo); (viii) TAM = Tamanho da empresa representado pelo log do Ativo Total da empresa no final do ano; e (ix) ENDIV = Índice de Endividamento da empresa no final do ano; (x) Sobre os asteriscos, *** $\mathrm{p}<0.01$, ** $\mathrm{p}<0.05$, e ${ }^{*} \mathrm{p}<0.10$;

Cabe ressaltar que todos os modelos se mostraram estatisticamente significativos a $1 \%$ (prob $>$ Chi2 inferior a 0.01), rejeitando a hipótese de que todos os coeficientes são 
conjuntamente iguais a zero. Os resultados apresentados estão baseados em uma interpretação que faz mais sentido, pois se dá em termos das chances, que podem ser calculadas por meio do antilogaritmo do coeficiente logit. Para os casos em que o coeficiente foi negativo, a razão de chances é obtida por meio do inverso do antilog do coeficiente (FÁVERO et al., 2009).

O primeiro modelo, como já mencionado, explora a influência da existência "bruta" de diferenças entre o lucro contábil e o fiscal, além das variáveis de controle. Foi possível observar que, com o acréscimo de um real de diferença entre o lucro contábil e o lucro fiscal, em média, a chance de a empresa propor o pagamento de dividendos não obrigatórios aumenta uma vez.

Observando os extremos, o modelo 2 verifica, a 5\% de alfa, que as chances também aumentam quando se trata de diferenças grandes negativas, contrariando o sinal esperado pela hipótese 1. Muito embora, considerando que a constante capta as informações omitidas acerca das variáveis dummies (interagidas) utilizadas no modelo e que ela apresentou um coeficiente negativo, se pode dizer que as chances de uma empresa distribuir dividendos adicionais reduzem em relação àquelas dos extremos. Assim, seria possível atender parcialmente à hipótese em questão. No entanto, a constante acaba por refletir outros efeitos não observáveis no modelo, impossibilitando tal conclusão.

O modelo 3 buscou capturar informações acerca das diferenças negativas, no intuito de atender à hipótese 2 da pesquisa (as chances de uma empresa com diferenças negativas propor distribuição de dividendos obrigatórios serão menores do que aquelas com diferenças positivas). Entretanto, não foi possível confirmar essa hipótese, devido ao fato de ambos os coeficientes não terem se mostrado significativos.

Dessa forma, os modelos seguintes buscaram minuciar essas diferenças a fim de conhecer as partes que influenciam na distribuição dos dividendos adicionais. Assim, os resultados do modelo 4 demonstraram, em um nível de significância de $1 \%$, que as chances aumentam em uma vez dado o acrescimento de 1 real de diferença permanente negativa.

De modo a corroborar esse resultado, o modelo 5, que abre as diferenças entre os extremos negativo e geral e ainda temporário e permanente, verificou a significância apenas para os coeficientes das diferenças permanentes. Só cabe fazer uma ressalva quanto às empresas que pertencem ao extremo negativo das diferenças permanentes negativas, pois, nesse caso, as chances diminuem com o acréscimo dessa diferença.

Sobre as variáveis de controle, para todos os modelos foi possível observar que empresas maiores têm mais chances de distribuir dividendos não obrigatórios, e que o aumento no endividamento das empresas diminui as chances de uma empresa propor tal distribuição, o que é coerente com a realidade das empresas, que, ao se endividarem, passam a ter como prioridade o pagamento da dívida, o que reduzirá o excesso de lucro, caso este ainda exista.

\section{Considerações Finais e Recomendações}

O objetivo deste estudo foi verificar em que nível a existência de diferenças entre o lucro contábil e o lucro fiscal (BTDs) impacta significativamente a probabilidade de uma empresa propor o pagamento de dividendos adicionais no mercado nacional, restrito ao processo de seleção da amostra. Ainda, investigou-se se o controle por grandes diferenças, ou

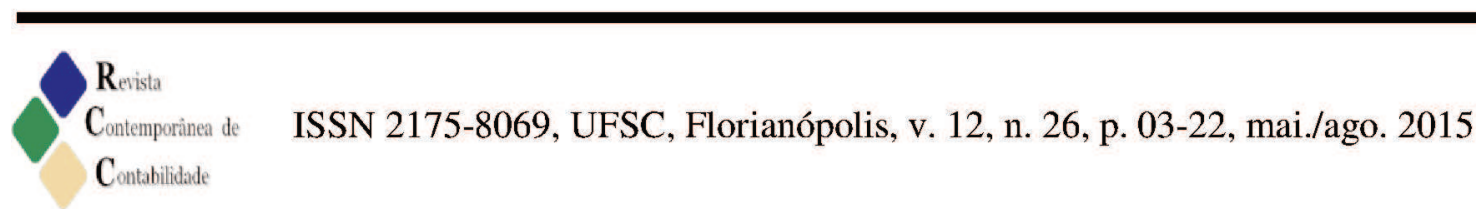


ainda quando se controla pelo sinal e pelos extremos dessas diferenças, influencia na propensão ao pagamento de dividendos não obrigatórios.

A investigação partiu de uma ressalva feita pelo seminal trabalho de Miller e Modigliani (1961), por meio do qual propuseram a teoria de irrelevância dos dividendos. A observação feita diz respeito à necessidade de investigação da manutenção da teoria em um ambiente com impostos, isto é, em um mercado imperfeito. Em meio a isso, com base em diversas evidências na literatura, buscou-se estudar especificamente a distribuição do excesso de lucro, que tem a evidenciação obrigatória pela Resolução n. 683/12 da CVM.

No mesmo trabalho, os autores propuseram uma explanação sobre o que chamaram de "efeito clientela", que Novis Neto e Saito (2002) simplificaram dizendo que ele é explicado pela suposta preferência de uma parte dos investidores em receber pagamentos de dividendos, em vez de ganhos de capital.

Assim, utilizando as BTDs como proxy para o impacto tributário na política de dividendos, foi necessário explorar as características que formam essas diferenças, isto é, diferenças temporárias e permanentes, no intuito de verificar qual provocaria impacto na propensão a distribuir os dividendos.

De maneira geral, os resultados não permitiram confirmar a hipótese $\left(\mathrm{H}_{1}\right)$ de que grandes diferenças (positivas e negativas) causariam redução na probabilidade de distribuição, pois, o coeficiente da variável que captura os valores do extremo negativo se mostrou significativo e positivo. Dessa forma, o não atendimento à hipótese acaba por revelar a preferência por distribuir dividendos mesmo quando o lucro fiscal sobrepuja o lucro contábil, isto é, parcialmente trata-se do efeito clientela, coerente com os achados de Novis Neto e Saito (2002).

Sobre a hipótese $\left(\mathrm{H}_{2}\right)$, de que as chances de uma empresa com diferenças negativas propor distribuição de dividendos obrigatórios serem menores do que aquelas com diferenças positivas, os resultados não foram claros. No entanto, os resultados dos modelos seguintes demonstraram que, em casos de diferenças temporárias, a probabilidade não é afetada, fazendo supor que os gestores reconhecem que tais diferenças serão revertidas em um tempo próximo, por isso, não seria viável distribuir um lucro que carrega essas BTDs. Cabe ressaltar que, para contrapor à amostra das empresas que propuseram a distribuição, o estudo se baseou em uma amostra aleatória. Mesmo assim, deve-se ter cautela sobre a generalização dos resultados.

Para futuras pesquisas, recomenda-se verificar a influência de fatores endógenos às diferenças causadas pela imposição fiscal na Contabilidade. Características setoriais talvez possam ajudar a explicar alguns resultados. No entanto, no intuito de manter a pequena amostra de empresas que atenderam ao evento de interesse, optou-se por extrair outra amostra com o mesmo número de observações por setor e por ano.

Outra opção seria talvez verificar a magnitude dessa distribuição, no sentido de enxergar as influências das BTDs no volume de dividendos adicionais distribuídos, trazer para discussão os ganhos de capital e, por fim, observar os impactos trazidos pelo advento da Lei n. 12.973/2014, que, embora não tenha afetado os dados utilizados na pesquisa, causará impacto nos anos subsequentes ao período amostral. 


\section{Referências}

AHARONY, J.; SWARY, I. Quarterly Dividend and Earnings Announcements and Stockholders' returns: an empirical analysis. The Journal of Finance, v. 35, n. 1, p. 1-12, $1^{\circ}$ mar. 1980.

ANTUNES, G. A.; LAMOUNIER, W. M.; BRESSAN, A. A. Análise do "efeito tamanho" nos retornos das ações de empresas listadas na Bovespa. Revista Contabilidade \& Finanças, v. 17, n. 40, p. 87-101, abr. 2006.

ASQUITH, P.; MULLINS, D. W., Jr. The impact of initiating dividend payments on shareholders' wealth. The Journal of Business, v. 56, n. 1, p. 77-96, $1^{\circ}$ jan. 1983.

BALL, R.; BROWN, P. An empirical evaluation of accounting income numbers. Journal of Accounting Research, v. 6, n. 2, p. 159, 1968.

BEAVER, W. H. The information content of annual earnings announcements. Journal of Accounting Research, v. 6, p. 67, 1968.

BEAVER, W. H. Perspectives on recent capital market research. The Accounting Review, v. 77, n. 2, p. 453-474, 2002.

BHATTACHARYA, S. Imperfect information, dividend policy, and "the bird in the hand" fallacy. Bell Journal of Economics, v. 10, n. 1, p. 259-270, 1979.

BLACK, F.; SCHOLES, M. The effects of dividend yield and dividend policy on common stock prices and returns. Journal of Financial Economics, v. 1, n. 1, p. 1-22, maio 1974.

BRASIL, Deliberação CVM n. 683, de 30 de agosto de 2012.

BRASIL, Lei n. 6.404, de 28 de dezembro de 2007.

BRASIL, Lei n. 9.249, de 26 de dezembro de 1996.

BRICKLEY, J. A. Shareholder wealth, information signaling and the specially designated dividend: an empirical study. Journal of Financial Economics, v. 12, n. 2, p. 187-209, ago. 1983.

DEANGELO, H.; DEANGELO, L.; SKINNER, D. J. Reversal of fortune dividend signaling and the disappearance of sustained earnings growth. Journal of Financial Economics, v. 40, n. 3, p. 341-371, mar. 1996. 
DECHOW, P.; GE, W.; SCHRAND, C. Understanding earnings quality: a review of the proxies, their determinants and their consequences. Journal of Accounting and Economics, v. 50, n. 2-3, p. 344-401, dez. 2010.

DRAKE, K. D. Does firm life cycle explain the relation between book-tax differences and earnings persistence? Rochester, NY: Social Science Research Network, 13 fev. 2013. Disponível em: <http://papers.ssrn.com/abstract=2217145>. Acesso em: 13 set. 2014.

FÁVERO, L. P. et al. Análise de dados. Modelagem multivariada para tomada de decisões. 7. ed. São Paulo: Elsevier, 2009.

FERREIRA, F. R. et al. Book-tax differences e gerenciamento de resultados no mercado de ações do Brasil. Revista de Administração de Empresas, v. 52, n. 5, p. 488-501, out. 2012.

GONEDES, N. J. Corporate signaling, external accounting, and capital market equilibrium: evidence on dividends, income, and extraordinary items. Journal of Accounting Research, v. 16, n. 1, p. 26, 1978.

HEALY, P. M.; PALEPU, K. G. Earnings information conveyed by dividend initiations and omissions. Journal of Financial Economics, v. 21, n. 2, p. 149-175, set. 1988.

JOOS, P., PRATT, J., YOUNG, D., 2000. Book-tax differences and the value relevance of earnings. Working Paper, Massachusetts Institute of Technology, Indiana University and Insead.

KALAY, A.; LOEWENSTEIN, U. Predictable events and excess returns: The case of dividend announcements. Journal of Financial Economics, v. 14, n. 3, p. 423-449, set. 1985.

LEVINE, D. M. Estatística - teoria e aplicações usando o Microsoft Excel. 6. ed. São Paulo. 2012: LTC, 2012.

LITZENBERGER, R. H.; RAMASWAMY, K. The effect of personal taxes and dividends on capital asset prices: Theory and empirical evidence. Journal of Financial Economics, v. 7, n. 2, p. 163-195, jun. 1979.

MICHAELY, R.; THALER, R. H.; WOMACK, K. L. Price Reactions to Dividend Initiations and Omissions: Overreaction or Drift? The Journal of Finance, v. 50, n. 2, p. 573-608, 1 jun. 1995.

MILLER, M. H.; MODIGLIANI, F. Dividend Policy, Growth, and the Valuation of Shares. The Journal of Business, v. 34, n. 4, p. 411-433, 1 out. 1961.

NOVIS NETO, J. A.; SAITO, R. Pagamentos de dividendos e persistência de retornos anormais das ações: evidência do mercado brasileiro. Revista de Administração, v. 38, n. 2 , p. 135-143, 2002. 
PENMAN, S. H. The predictive content of earnings forecasts and dividends. The Journal of Finance, v. 38, n. 4, p. 1181-1199, $1^{\circ}$ set. 1983.

SILVA, E. B. G. DA. A relevância do dividendo adicional proposto. $\mathbf{1 0}^{\circ}$ Congresso USP de Iniciação Científica em Contabilidade, n. 10, 2013.

WATTS, R. The information content of dividends. The Journal of Business, v. 46, n. 2, p. 191-211, $1^{\circ}$ abr. 1973.

WATTS, R. L.; ZIMMERMAN, J. L. Towards a positive theory of the determination of accounting standards. The Accounting Review. 53, n. 1, p. 112-134, jan. 1978. 